\title{
The visualisation of vitreous using surface modified poly(lactic-co-glycolic acid) microparticles
}

\author{
David YS Chau, ${ }^{1}$ Naing L Tint, ${ }^{1,2}$ Russell J Collighan, ${ }^{3}$ Martin Griffin, ${ }^{3}$ \\ Harminder S Dua, ${ }^{2}$ Kevin M Shakesheff, ${ }^{1}$ Felicity R A J Rose ${ }^{1}$
}

\begin{abstract}
${ }^{1}$ Division of Drug Delivery and Tissue Engineering, Wolfson Centre for Stem Cells, Tissue Engineering and Modelling, Centre for Biomolecular

Sciences, School of Pharmacy, University of Nottingham, University Park, Nottingham, UK ${ }^{2}$ Division of Ophthalmology and Vision Sciences, University of Nottingham, Queen's Medical Centre, UK

${ }^{3}$ Life and Health Sciences, Aston University, Aston Triangle, Birmingham, UK
\end{abstract}

\section{Correspondence to}

Dr Felicity R A J Rose, Wolfson Centre for Stem Cells, Tissue Engineering and Modelling, Centre for Biomolecular

Sciences, School of Pharmacy, University of Nottingham, University Park, Nottingham NG7 2RD, UK;

felicity.rose@nottingham.ac.uk

Accepted 20 December 2009

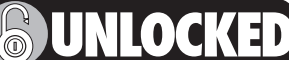

This paper is freely available online under the BMJ Journals unlocked scheme, see http:// bjo.bmj.com/site/about/ unlocked.xhtml.

\section{ABSTRACT}

Aims To demonstrate the potential use of in vitro poly (lactic-co-glycolic acid) (PLGA) microparticles in comparison with triamcinolone suspension to aid visualisation of vitreous during anterior and posterior vitrectomy.

Methods PLGA microparticles (diameter 10-60 $\mu \mathrm{m}$ ) were fabricated using single and/or double emulsion technique(s) and used untreated or following the surface adsorption of a protein (transglutaminase). Particle size, shape, morphology and surface topography were assessed using scanning electron microscopy (SEM) and compared with a standard triamcinolone suspension. The efficacy of these microparticles to enhance visualisation of vitreous against the triamcinolone suspension was assessed using an in vitro set-up exploiting porcine vitreous.

Results Unmodified PLGA microparticles failed to adequately adhere to porcine vitreous and were readily washed out by irrigation. In contrast, modified transglutaminase-coated PLGA microparticles demonstrated a significant improvement in adhesiveness and were comparable to a triamcinolone suspension in their ability to enhance the visualisation of vitreous. This adhesive behaviour also demonstrated selectivity by not binding to the corneal endothelium.

Conclusion The use of transglutaminase-modified biodegradable PLGA microparticles represents a novel method of visualising vitreous and aiding vitrectomy. This method may provide a distinct alternative for the visualisation of vitreous whilst eliminating the pharmacological effects of triamcinolone acetonide suspension.

\section{INTRODUCTION}

Posterior capsule rupture resulting in vitreous prolapse is a common complication during cataract extraction. The incidence of posterior capsule rupture after phacoemulsification ranges from $1.41 \%$ to $5.1 \%{ }^{1}$ A thorough anterior vitrectomy must be performed to reduce the incidence of complications such as elevated intraocular pressure, cystoid macular oedema, retinal detachment, eccentric pupil and endophthalmitis. ${ }^{2}$ The surgeons' ability to undertake an adequate anterior vitrectomy is often made difficult by the inability to adequately visualise vitreous in the anterior chamber under a surgical microscope and, for this reason, a substance is needed to enhance the visualisation of the vitreous.

Triamcinolone acetonide, a particulate steroid suspension, has been successfully utilised in visualising vitreous during anterior vitrectomy. ${ }^{3}$ It is rinsed and diluted 1:10 prior to use. ${ }^{3}$ Nevertheless, animal studies have shown the potential for endothelial damage and toxicity with its use. ${ }^{5} 6$ Moreover, the use of triamcinolone within the anterior chamber predisposes the eye to undesired and uncontrolled intraocular delivery of a steroid. As such, there is a risk of elevated post-operative intraocular pressure and steroid-induced glaucoma, even though removal of the agent is attempted at the time of surgery. ${ }^{78}$

More recently dyes and particulate substances have been proposed as an alternative to the use of triamcinolone. $^{7-12}$ However, no extensive longterm experimental studies on the safety of these stains as intraocular dyes have been performed and there are conflicting reports on their safety. ${ }^{711} 12$

Poly(lactic-co-glycolic acid) (PLGA) is a biodegradable polymer that is used extensively in biomedical applications, including in the eye. ${ }^{13}$ In addition, growth factor- and/or drug-incorporated PLGA microparticles have also been exploited as drug delivery vehicles to the posterior segment with in vivo rabbit studies demonstrating the biocompatibility of intravitreally administered PLGA microparticles. ${ }^{14-16}$ Furthermore, PLGA can be extensively modified by various modalities that can render it more hydrophilic and enhance its biocompatibility. ${ }^{17}$

In this present study, we demonstrate the effectiveness, in vitro, of surface-modified PLGA microparticles in allowing adequate visualisation of vitreous.

\section{MATERIALS AND METHODS}

\section{Manufacture of PLGA microparticles}

PLGA (PLGA 85:15 DL; High IV; molecular weight $118 \mathrm{kDa}$ ) (Lakeshore Biomaterials, Birmingham, Alabama, USA) microparticles were fabricated using a single oil-in-water $(\mathrm{O} / \mathrm{W})$ emulsion technique as previously described. ${ }^{18}$ Briefly, $1.0 \mathrm{~g}$ of PLGA polymer was dissolved in $5 \mathrm{ml}$ dichloromethane (DCM) (Fisher Scientific, Loughborough, UK) overnight at room temperature in a glass scintillation phial. Five millilitres of $0.3 \%$ (weight/ volume) poly(vinyl alcohol) (PVA; molecular weight $13000-23000 \mathrm{kDa} ; 87-89 \%$ hydrolysed) (Sigma-Aldrich, Poole, UK) was then slowly added to the phial before the solution was emulsified using a Chiltern Scientific VM20 vortex mixer, scale 5 setting, for $45 \mathrm{~s}$ (Chiltern Scientific Instruments, Wendover, UK). This primary O/W emulsion was poured into a $0.3 \%$ (weight/volume) PVA solution water-bath and stirred constantly overnight to allow the solvent to evaporate. Microparticles were then sieved and separated using a Retsch AS200 sieve shaker (Retsch UK Ltd, Leeds, UK). 
Oil-Red-O (Red O dye (Solvent Red 27; (1-([4-(xylylazo)xylyl] azo)-2-naphthol)) and/or Fast-Green-FCF (both from SigmaAldrich) were incorporated into the microparticles using the aforementioned $\mathrm{O} / \mathrm{W}$ single emulsion technique. One hundred microlitres of the dye was thoroughly mixed with the $5 \mathrm{ml}$ PLGA/DCM solution to form the primary emulsion and processed as described above.

\section{Size profiling and distribution}

The size distribution of the pre-fractionated microparticles was determined by laser diffraction. Samples were dispersed in $4 \mathrm{ml}$ of distilled water within the chamber of a Coulter LS230 particle size analyser (Beckman Coulter, High Wycombe, UK) under moderate stirring (default speed 3 setting) to the required concentration as indicated by the in-built display software. Particle size ranges were defined using PSS-Duke standards (Polymer Standard Service, Kromatek Ltd, Dunmow, UK). Particle size distribution was then determined as a function of the particle diffraction using the Coulter software (version 2.11a) and plotted as a function of the percentage of distribution volume.

\section{Transglutaminase isolation and purification}

The microbial transglutaminase (mTG; isolated from Streptoverticillium mobaraense) used throughout this investigation was sourced from the commercially available product, Activa WM (Ajinomoto Corporation Inc., Tokyo, Japan). Tissue transglutaminase (mammalian transglutaminase; tTG) was isolated and purified from guinea pig livers as previously described. ${ }^{19}$

\section{Surface adsorption of proteins onto microparticles}

Microparticles were pre-wetted with serum-free Dulbecco's modified Eagle's medium (DMEM) for $24 \mathrm{~h}$ in a humidifiedatmosphere incubator at $37^{\circ} \mathrm{C}$ and $5 \%$ (volume/volume) $\mathrm{CO}_{2}$ in air prior before being coated with the appropriate protein/ enzyme (ie, bovine serum albumin (BSA) control, mTG or tTG) using a standard surface adsorption protocol. Microparticles (30 mg) were incubated overnight with $1 \mathrm{ml}$ of either $1 \mathrm{mg} / \mathrm{ml}$ BSA, mTG or tTG solution on a Stuart SRT6 roller mixer (VWR International Ltd, Lutterworth, UK) (default settings) in a humidified-atmosphere incubator at $37^{\circ} \mathrm{C}, 5 \%$ (volume/ volume) $\mathrm{CO}_{2}$ in air. Following the coating procedure, samples were washed once with $10 \mathrm{mmol} / \mathrm{l}$ EDTA and then with PBS, $\mathrm{pH} 7.4$, twice, before immediate use.

Samples exploiting the active-site directed inhibitor termed 'R281' (a synthetic carboxybenzoyl-glutaminyl-glycine analogue, developed in-house) were used as negative controls to the crosslinking activity of the enzyme (pre-treatment for $1 \mathrm{~h}, 500 \mu \mathrm{mol} / \mathrm{l}$, at $37^{\circ} \mathrm{C}$, followed by dialysis in phosphate buffered saline (PBS), pH 7.4) as previously described. ${ }^{19}{ }^{20}$ Non-crosslinking tTG enzyme (ie, without the addition $\mathrm{CaCl}_{2}$ ) was also used as a negative control for catalytic activity.

\section{Visualisation of vitreous}

The efficacy of these microparticles to enhance the visualisation of vitreous against triamcinolone suspension (triamcinolone acetonide solution (Kenalog; $40 \mathrm{mg} / \mathrm{ml}$ ) (ER Squibb and Sons Ltd, Uxbridge, UK) was assessed using a simple in vitro set-up exploiting porcine vitreous (Local abattoir, Nottingham, UK). Cornea, iris and lens were dissected allowing adequate access to the vitreous cavity. The vitreous was grasped using microtoothed forceps before being cut with Westcott scissors. The vitreous was placed in $2 \mathrm{ml} \mathrm{PBS}, \mathrm{pH} \mathrm{7.4}$, in a 24 well plate. Microparticles and/or triamcinolone suspension were then added, using a Rycroft cannula, to the vitreous. Samples were then transferred to adjacent PBS-filled wells using a Pasteur pipette and thoroughly agitated. Consecutive washes were performed using this method before samples were viewed using a Leica MZ16F stereomicroscope and images captured using the in-built camera.

\section{RESULTS}

\section{The fabrication of PLGA microparticles}

PLGA microparticles were fabricated using the single O/W technique and analysed by SEM and stereomicroscopy for both morphology and topography, as summarised in figure 1 . Microparticles manufactured using this technique were spherical and possessed smooth surface characteristics with a typical size distribution shown in figure 2. These microparticles could also
Figure 1 Scanning electron microscope (SEM) and brightfield microscopy images of triamcinolone acetonide suspension and poly(lactic-coglycolic acid) (PLGA) microparticles. Lyophilised triamcinolone solution and 20-100 $\mu \mathrm{m}$ PLGA microparticles were mounted on aluminium SEM stubs, goldcoated under an argon atmosphere before being imaged using a JOEL $6060 \mathrm{LV}$ variable pressure SEM operating at an accelerating voltage of $10 \mathrm{kV}$ at the corresponding magnification $(A, B$ and $C$, $D$, respectively). Brightfield images of $1 \mathrm{mg} / \mathrm{ml}$ triamcinolone solution and PLGA microparticles, in phosphate buffered saline (PBS), were achieved using a Leica DM-IRB/E inverted microscope and captured using the in-built imaging software ( $\mathrm{C}$ and $\mathrm{F}$, respectively).
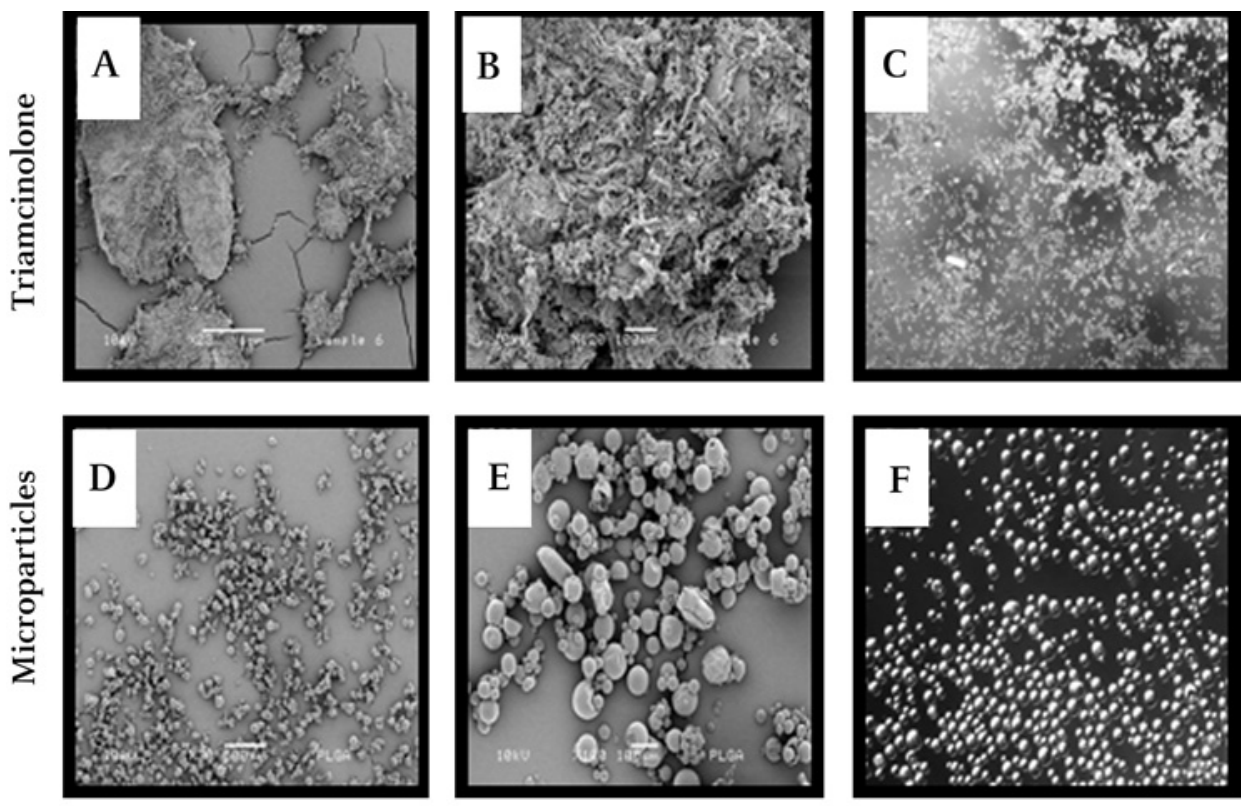

SEM 


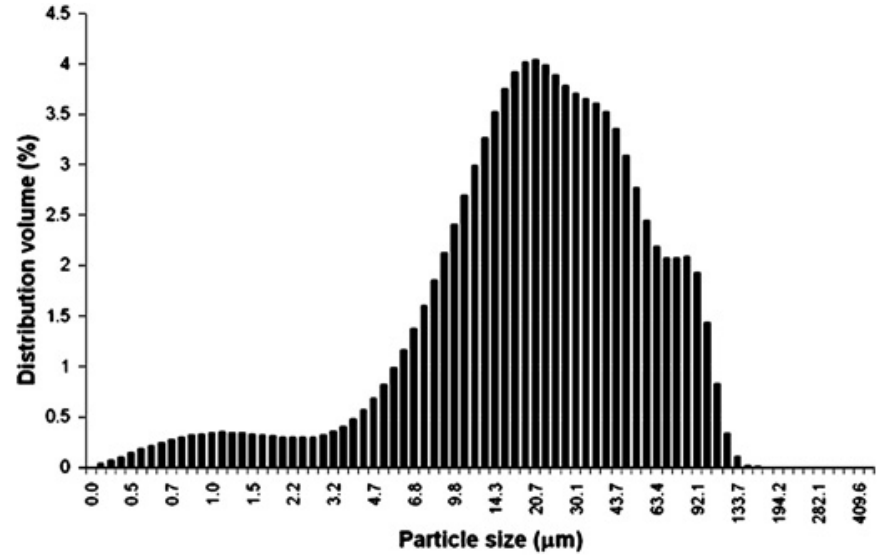

Figure 2 Size distribution of fabricated poly(lactic-co-glycolic acid) (PLGA) microparticles. Microparticles were dispersed in $4 \mathrm{ml}$ of distilled water within the chamber of a Coulter LS230 particle size analyser under moderate stirring. Particle size distribution was then determined as a function of the particle diffraction using the Coulter software (version 2.11a) and plotted, as shown above, as a function of the percentage of distribution volume.

be further fractionated within the $10-60 \mu \mathrm{m}$ size range, which was comparable to the particulate size found in the triamcinolone acetonide suspension $(<50 \mu \mathrm{m})$. Moreover, on comparison to the triamcinolone acetonide suspension, which demonstrated a random-sized particulate mixture, these microparticles were fabricated with consistent pre-defined parameters and associated characteristics (ie, uniform shape, size and surface topography within a defined distribution). The successful incorporation of 'molecules-of-interest' was also demonstrated by the incorpo- ration of selective dye reagents (ie, Oil-Red-O and Fast-GreenFCF). In addition, further modification of these microparticles was achieved via the surface adsorption of proteins, namely TG or BSA.

\section{Enhancing visualisation of vitreous}

Sample vitreous, once wetted, is extremely difficult to visualise, as seen in figure $3 \mathrm{~A}$. In contrast, following the addition of triamcinolone acetonide, the vitreous becomes more obvious and easier to identify in solution (ie, irrigation wash during typical ophthalmic surgery) as demonstrated in figure 3B. Even after washing in PBS, the vitreous exposed to triamcinolone can still be visualised. Control PLGA microparticles (ie, not surface modified), coloured red via the incorporation of Oil-Red-O dye to aid visualisation during manipulation in these experiments, demonstrated no adhesion to the vitreous and were almost completely removed following consecutive washes with PBS (figure 4A). In comparison, PLGA microparticles treated by the surface adsorption of $\mathrm{mTG}$ (PLGA-mTG-MP), demonstrated a significant increase and ability to adhere to the vitreous and maintained complete adhesion even following extensive consecutive washing steps (figure 4B,C). On comparing a normalised quantity (weight for weight in a fixed volume) of triamcinolone acetonide and PLGA microparticles, it can be seen that the enhanced visualisation effect of the microparticles was comparable to the triamcinolone suspension (comparing figure $4 \mathrm{C}$ with figure $3 \mathrm{~B}$ ). Control microparticles that were pretreated/surface adsorbed with BSA (PLGA-BSA-MP) demonstrated no adhesive properties at all and appeared to be unable to localise around the vitreous during the addition step. PLGA-BSAMPs were almost completely removed during the initial washing step with PBS (figure 5A). Verification of the adhesive nature
Figure 3 Visualisation of vitreous using triamcinolone solution. The comparative visualisation of $(A)$ control vitreous and (B) triamcinolone acetonide-treated vitreous were visualised using a Leica MZ16F stereomicroscope immediately following the addition of phosphate buffered saline (PBS) or triamcinolone (left) and following consecutive washes with PBS solution (right). Images were captured using the in-built software and shown at $1.6 \times$ magnification.
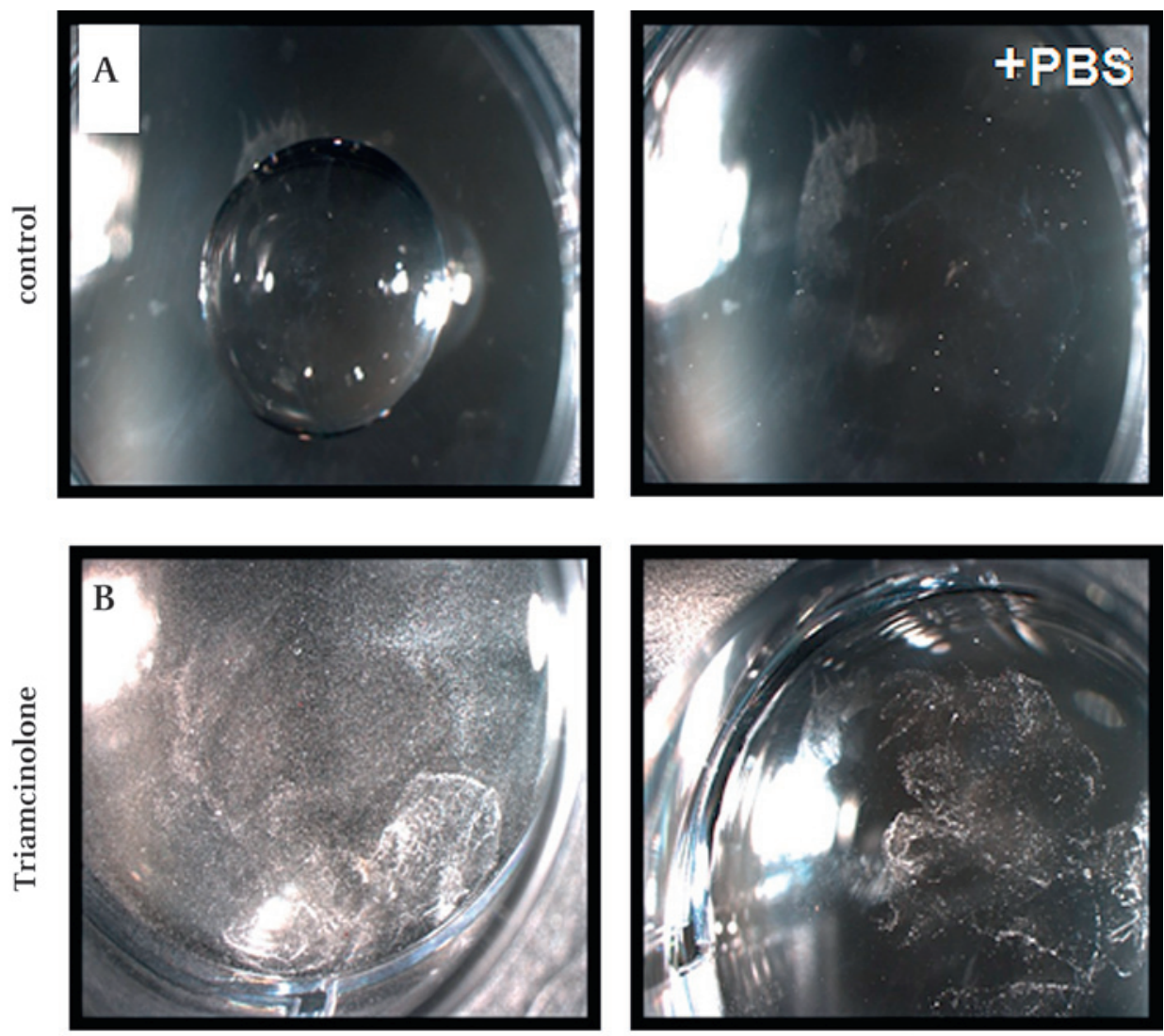

addition

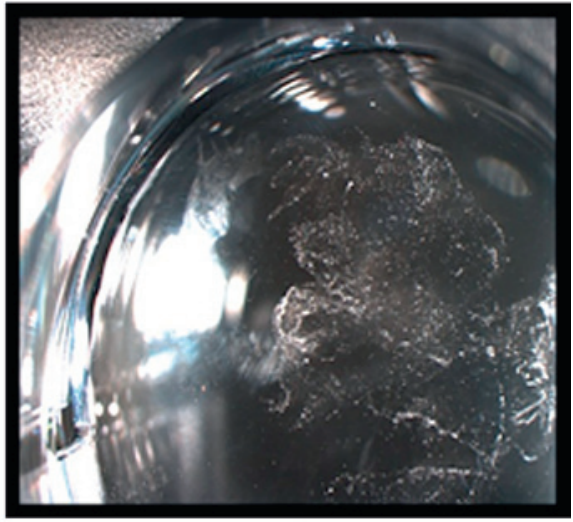

wash 2 
Figure 4 Visualisation of vitreous using poly(lactic-co-glycolic acid) (PLGA) microparticles. The comparative visualisation of vitreous following incubation with $(A)$ untreated Oil-Red-0 incorporated PLGA microparticles, (B) Oil-Red-0 incorporated microbial transglutaminase-treated PLGA microparticles, (C) microbial transglutaminase-treated PLGA microparticles (no Oil-Red-0). Microparticles used were in the 20-100 $\mu \mathrm{m}$ diameter range and samples visualised using a Leica MZ16F stereomicroscope immediately following the addition (left) and after two consecutive washes (middle and right) software and shown at $1.6 \times$ magnification.

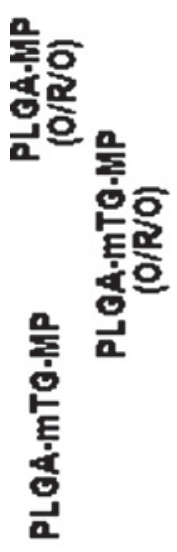

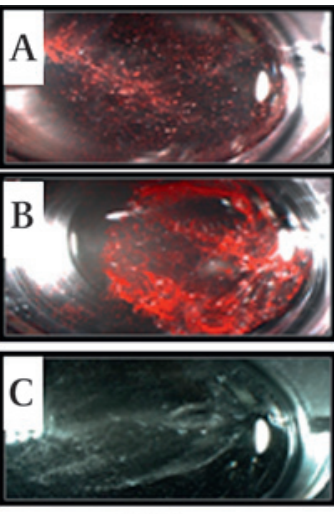

addition

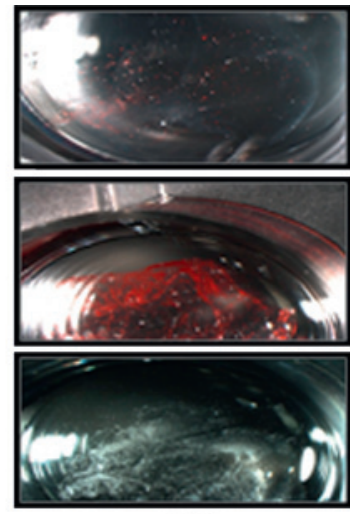

wash 1

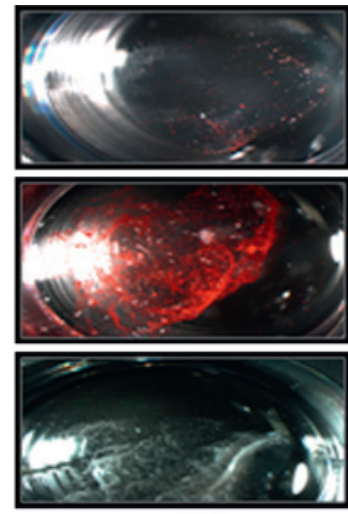

wash 2 of the PLGA-mTG-MPs was further investigated using the R281-inhibited control samples of the mTG (figure 5B). A similar behaviour was observed using the inactivated mammalian form of the enzyme (ie, tTG without addition of $\mathrm{CaCl}_{2}$ ) as seen in figure $5 \mathrm{C}$.

\section{Selective adhesiveness of PLGA microparticles}

The adhesion of (Oil-Red-O incorporated) PLGA-mTG-MP to the sample vitreous was found to be stable even after several extensive washing steps (more than seven washes with PBS). However, the ability of these microparticles to adhere to other tissues was found to be selective. PLGA-TG-MP was unable to adhere to the corneal endothelium or iris and were completely removed during the first washing step (not shown) as would be desired in surgical applications to minimise endothelial damage or compromise.

\section{DISCUSSION}

Thorough removal of the vitreous is essential in cases of vitreous prolapsed in to the anterior chamber during cataract surgery and in retinal detachment and proliferative diabetic retinopathy requiring pars plana vitrectomy (PPV). The vitreous, though transparent in nature, may be visualised by light scatter such that there is an increase in the angle between illumination and viewing. In the setting of PPV, this can be achieved via the use of a fibre optic light pipe. However, the visualisation of the anterior vitreous and vitreous base using co-axial illumination from an operating microscope can be problematic. ${ }^{21}$ An agent that permits visualisation of the vitreous is therefore required during surgery and is of particular importance to ophthalmic surgeons in training. 9 "The current, commonly used, method exploits triamcinolone acetonide to aid the visualisation of vitreous during such situations. However, not only do the tedious preparation steps for triamcinolone limit its use during surgery, but the direct addition and possibility of residual steroid localised within the eye poses additional risks to the patient. ${ }^{3}$ Furthermore, in vivo models have also demonstrated endothelial damage and toxicity following use of intracameral triamcinolone. ${ }^{56}$ Hence, in this study, we present a novel methodology of visualising vitreous using microparticles fabricated from PLGA with the aim of minimising the risk of introducing endogenous steroid agents or the toxicity issues associated with certain ophthalmological dyes.

The biodegradable polymer, PLGA, has been used extensively for many tissue engineering and drug delivery applications. An important feature of this polymer is that it gradually breaks down within an aqueous environment and degrades into water-soluble, non-toxic, by-products. These degradation products can then be cleared by the body through metabolic pathways and hence are eliminated from the implant site. ${ }^{22}$ In this investigation, we
Figure 5 Visualisation of vitreous using modified poly(lactic-co-glycolic acid) (PLGA) microparticles. The comparative visualisation of vitreous following incubation with (A) FastGreen-FCF incorporated bovine serum albumin (BSA)-treated PLGA microparticles and (B) Oil-Red-O incorporated microbial transglutaminase-treated PLGA microparticles. Transglutaminase crosslinking activity was inhibited with the active-site directed inhibitor, R281, prior to adsorption on the microparticle surface. (C) Oil-Red-0 incorporated mammalian transglutaminase-treated PLGA microparticles. Microparticles used were in the $20-100-\mu \mathrm{m}$ range and samples visualised using a Leica MZ16F stereomicroscope following the addition (left) and after consecutive washes (middle and right) of the microparticles with phosphate buffered saline (PBS). Images were captured using the in-built software and shown at $1.6 \times$ magnification.

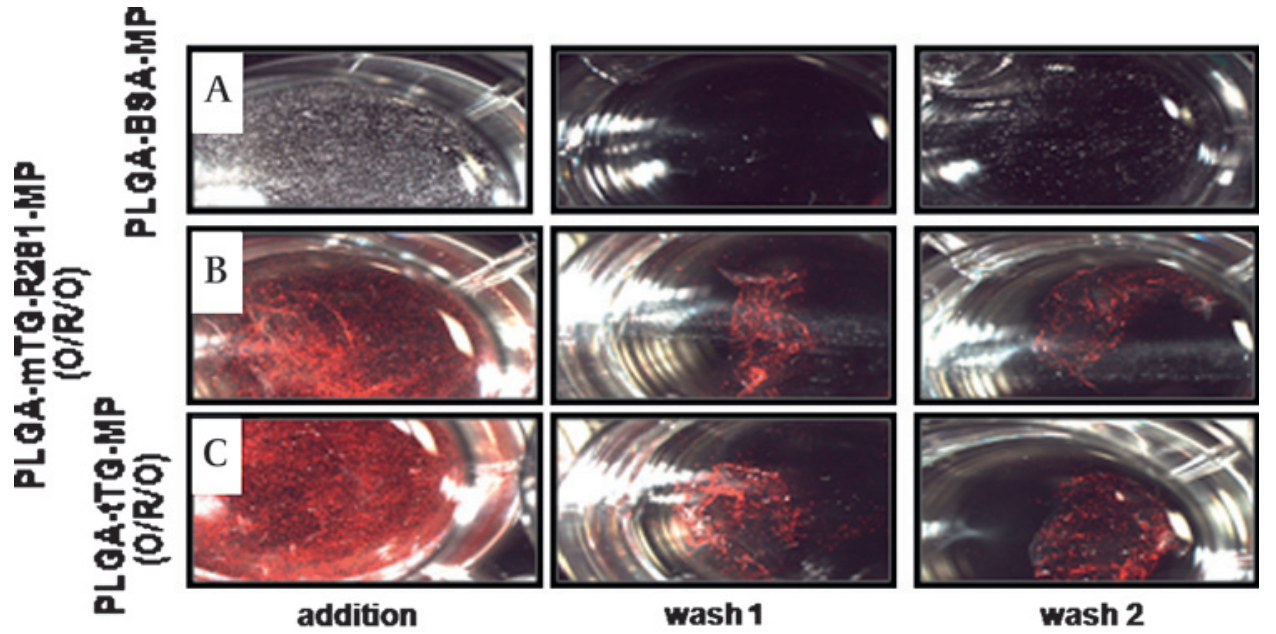


utilised the PLGA polymer in the form of microparticles solely as a visualisation tool. However, these microparticles could to be exploited in the future as a mode of targeted drug delivery.

Within this study, the characteristics (ie, particle shape and size) of these fabricated microparticles were shown to be comparable to the insoluble particulates found in the triamcinolone acetonide suspension which, based on the integration of the crystals into loosely organised collagen matrices, allows the visualisation of the vitreous. ${ }^{23}$ However, in contrast, unmodified PLGA microparticles were unable to sufficiently adhere to vitreous without prior treatment, in this case, with the enzyme TG. TG, commonly referred to as a 'biological glue', has been reported extensively to act as a biocatalyst, to enhance cell adhesion, to promote surface modifications and to increase the biocompatibility of many tissue engineering scaffolds without inducing any significant immune response. ${ }^{24-28}$ Previous experiments have already demonstrated the potential clinical use of TG as a novel cartilage-cartilage biological glue. ${ }^{29}$ As such, it was hypothesised that the pre-surface adsorption of the PLGA microparticles with TG would enhance its adhesiveness and aid the visualisation of vitreous. Such a system could be easily exploited during surgery.

This study showed that TG-treated microparticles do indeed adhere to the vitreous in such a manner that is comparable to triamcinolone acetonide suspension. Moreover, this adhesiveness was shown to be selective and the microparticles would only adhere to the vitreous and not to the other anterior chamber structures (ie, the corneal endothelium and iris). It is hypothesised that this behaviour may be based upon the ability of the enzymes to bind to peptide sequences up to 12 residues long, with collagen being a good substrate. ${ }^{19} 3031$ Further confirmation of this effect being solely dependent on the pre-surface adsorption of the TG can be seen in comparison with BSA-treated control microparticles. Above all, repeated washing of the microparticles could not dislodge and/or facilitate their removal from the vitreous and hence, they would be ideal for application as a visualisation tool to aid the removal of prolapsed vitreous, which otherwise is virtually invisible. In addition, similar and corresponding effects between the microbial and mammalian versions of the enzyme have been demonstrated. This means that in vivo applications may be potentially considered with minimal immunological response. In addition, the incorporation of selective dyes (eg, Oil-Red-O and Fast-Green-FCF) demonstrates the possibility of entrapping anti-microbials/drugs and/or other molecules of interest within these microparticles to facilitate and enhance healing during or following surgery. ${ }^{32}$ Hence, the presence of any residual microparticles may not be a disadvantage (compared with the presence of steroids or their precursors) but, in fact, may be desired in specific applications.

\section{CONCLUSIONS}

Herein, we report a novel method of visualising vitreous and aiding anterior vitrectomy by exploiting the use of biodegradable PLGA microparticles. This method may provide a distinct alternative to the use of triamcinolone acetonide suspension and other common surgical dyes without the associated pharmacological and/or toxicity effects. However, extensive in vivo studies are required to further investigate the efficacy and safety profile of this novel system prior to potential human use.

Acknowledgements We thank to the Laboratory of Biophysics and Surface Analysis (LBSA), School of Pharmacy, University of Nottingham, for use of the Coulter Counter, and Christine Grainger-Boultby for help with the SEM. Author NLT acknowledges the Medical Research Council for its funding via the Discipline Hopping Fellowship scheme.
Contributors DYSC and NLT contributed equally to the work and should be considered as joint first authors.

Funding MRC Discipline Hopper Grant.

Competing interests KMS is affiliated with Regentec Ltd (Nottingham, UK) as Chie Scientific Director. MG is affiliated with Xlink Ltd (Nottingham, UK) as Board Member Aside from the aforementioned, the authors have no relevant affiliations or financial involvement with any organisation or entity with a financial interest in or financial conflict with the subject matter or materials discussed in the manuscript. This includes employment, consultancies, honoraria, stock ownership or options, expert testimony, grants or patents received or pending, or royalties.

Provenance and peer review Not commissioned; externally peer reviewed.

\section{REFERENCES}

1. Johnston RL, Taylor H, Smith R, et al. The cataract National Dataset Electronic Multi-centre Audit of 55567 Operations: variation in posterior capsule rupture rates between surgeons. Eye (Lond) 2009. Aug 14. [Epub ahead of print]. doi:10.1038/ eye.2009.195.

2. Pingree MF, Crandall AS, Olson RJ. Cataract surgery complications in 1 year at an academic institution. J Cataract Refract Surg 1999;25:705-8.

3. Burk SE, Da Mata AP, Snyder ME, et al. Visualizing vitreous using Kenalog suspension. J Cataract Refract Surg 2003;29:645-51.

4. Yamakiri K, Uchino E, Kimura K, et al. Intracameral triamcinolone helps to visualize and remove the vitreous body in anterior chamber in cataract surgery. Am J Ophthalmol 2004;138:650-2.

5. Oh JY, Wee WR, Lee JH, et al. Short-term effect of intracameral triamcinolone acetonide on corneal endothelium using the rabbit model. Eye 2007; 21:812-18.

6. Chang YS, Tseng SY, Tseng SH, et al. Triamcinolone acetonide suspension toxicity to corneal endothelial cells. J Cataract Refract Surg 2006; 32:1549-55.

7. Kaji Y, Hiraoka $T$, Okamoto $F$ et al. Comparison of triamcinolone acetonide, 11 -deoxycortisol and other lipid formulae for the visualization of vitreous body in the anterior chamber after posterior capsule rupture in animal models. Acta Ophthalmol 2008:86:97-102.

8. Luo YH, Poole TR, Griffiths MF. Safe and effective visualisation of vitreous in the anterior chamber with intracameral fluorescein to facilitate its complete removal. Eve 2006;20:951-5.

9. Haritoglou C, Strauss R, Priglinger SG, et al. Delineation of the vitreous and posterior hyaloid using bromophenol blue. Retina 2008;28:333-9.

10. Yamashita T, Sakamoto T, Yamakiri K, et al. Polylactic acid for visualizing the vitreous body during vitrectomy. Invest Ophthalmol Vis Sci 2007;48: 3277-82.

11. Rodrigues EB, Costa EF, Penha FM, et al. The use of vital dyes in ocular surgery. Surv Ophthalmol 2009:54:576-617.

12. Collaer N, Stalmans $\mathrm{P}$. Which colour suits the vitreoretinal surgeon? Br J Ophthalmol 2007;91:1101-2

13. Hasirci V. Lewandrowski K, Gresser JD, Wise DL, Trantolo DJ. Versatility of biodegradable biopolymers: degradability and an in vivo application. J Biotechnol 2001;86:135-50.

14. Giordano GG, Chevez-Barrios $P$, Refojo MF, et al. Biodegradation and tissue reaction to intravitreous biodegradable poly(D, L-lactic-co-glycolic)acid microspheres. Curr Eye Res 1995; 14:761-8.

15. Wang M, Zhang JJ, Jackson TL, et al. Safety and efficacy of intracapsular tranilas microspheres in experimental posterior capsule opacification. J Cataract Refract Surg 2007; 33:2122-8

16. Herrero-Vanrell R, Refojo MF. Biodegradable microspheres for vitreoretinal drug delivery. Adv Drug Deliv Rev 2001;52:5-16.

17. Park GE, Pattison MA, Park K, et al. Accelerated chondrocyte functions on $\mathrm{NaOH}$-treated PLGA scaffolds. Biomaterials 2005:26:3075-82.

18. Bible E, Chau DY, Alexander MR, et al. Attachment of stem cells to scaffold particles for intra-cerebral transplantation. Nat Protoc 2009:4:1440-53.

19. Chau DY, Collighan RJ, Verderio EA, et al. The cellular response to transglutaminasecross-linked collagen. Biomaterials 2005;26:6518-29.

20. Skill NJ, Johnson TS, Coutts IG, et al. Inhibition of transglutaminase activity reduces extracellular matrix accumulation induced by high glucose levels in proximal tubular epithelial cells. J Biol Chem 2004;279:47754-62.

21. Veckeneer $\mathbf{M}$, Wong D. Visualising vitreous through a modified trans-scleral illumination by maximising the Tyndall effect. Br J Ophthalmol 2009;93:268-70.

22. Sokolsky-Papkov $\mathbf{M}$, Agashi K, Olaye A, et al. Polymer carriers for drug delivery in tissue engineering. Adv Drug Deliv Rev 2007:59:187-206.

23. Couch SM, Bakri SJ. Use of triamcinolone during vitrectomy surgery to visualize membranes and vitreous. Clin Ophthalmol 2009:3:41-7.

24. Yokoyama K, Nio N, Kikuchi Y. Properties and applications of microbial transglutaminase. Appl Microbiol Biotechnol 2004;64:447-54.

25. Griffin M, Casadio R, Bergamini CM. Transglutaminases: nature's biological glues. Biochem J 2002;368:377-96.

26. Verderio $\mathbf{E}$, Coombes A, Jones RA, et al. Role of the cross-linking enzyme tissue transglutaminase in the biological recognition of synthetic biodegradable polymers. J Biomed Mater Res 2001;54:294-304. 
27. Lorand L, Graham RM. Transglutaminases: crosslinking enzymes with pleiotropic functions. Nat Rev Mol Cell Biol 2003:4:140-56.

28. Garcia Y, Wilkins B, Collighan RJ, et al. Towards development of

a dermal rudiment for enhanced wound healing response. Biomaterials 2008; 29:857-68.

29. Jurgensen K, Aeschlimann D, Cavin V, et al. A new biological glue for cartilagecartilage interfaces: tissue transglutaminase. J Bone Joint Surg Am 1997; 79:185-93.
30. Hitomi K, Kitamura M, Sugimura Y. Preferred substrate sequences for transglutaminase 2: screening using a phage-displayed peptide library. Amino Acids 2009;36:619-24.

31. Sugimura Y, Yokoyama K, Nio N, et al. Identification of preferred substrate sequences of microbial transglutaminase from Streptomyces mobaraensis using a phage-displayed peptide library. Arch Biochem Biophys 2008:477:379-83.

32. Wu MC, Bhandari A. Managing the broken capsule. Curr Opin Ophthalmol 2008; 19:36-40. 